
\title{
25 Research Square \\ The Prognostic Value of Cardiac Troponin T in Different Age Groups of Traumatic Brain Injury Patients
}

\section{Ruoran Wang}

.Department of Critical Care Medicine, West China Hospital, Sichuan University, Chengdu, Sichuan province, China

\section{Min He}

.Department of Critical Care Medicine, West China Hospital, Sichuan University, Chengdu, Sichuan province, China

\section{Yan Kang ( $\boldsymbol{\sigma}$ kangyan@scu.edu.cn )}

.Department of Critical Care Medicine, West China Hospital, Sichuan University, Chengdu, Sichuan province, China https://orcid.org/0000-0001-8357-9561

\section{Research article}

Keywords: Troponin T, traumatic brain injury, marker, prognosis

Posted Date: August 6th, 2020

DOI: https://doi.org/10.21203/rs.3.rs-50058/v1

License: (1) (i) This work is licensed under a Creative Commons Attribution 4.0 International License. Read Full License 


\section{Abstract \\ Background}

The cardiac dysfunction has been confirmed as a common non-neurological complication and associated with increased mortality in traumatic brain injury (TBI) patients. As a biological marker of cardiac injury, the cardiac troponin T (TnT) has been verified correlated with outcome of some nontraumatic brain injury patients. However, the prognostic value of TnT in TBI patients has not been clearly illustrated. We designed this study to explore the association between TnT and outcome of TBI patients in different age subgroups.

\section{Methods}

Patients diagnosed with TBI in a prospective critical care database were eligible for this study. Univariate logistic regression analysis was firstly performed to explore the relationship between included variables and mortality. Then, the real effect of TnT on outcome of different age subgroups was analyzed by multivariate logistic regression analysis adjusting the confounding effects of other significant risk factors. Finally, we draw receiver operating characteristic (ROC) curves to evaluate the prognostic value of TnT in different age groups of TBI patients.

\section{Results}

520 patients were included in this study with mortality rate of $20.2 \%$. There were $112(21.5 \%)$ non-elderly patients (age $<65)$ and $408(78.5 \%)$ elderly patients (age $\geq 65)$. Non-survivors had higher percentage of previous acute myocardial infarction $(p=0.019)$ and pupil no-reaction $(p=0.028 ; p=0.011)$ than survivors. Survivors had higher GCS $(p<0.001)$ and lower TnT than non-survivors $(p<0.001)$. TnT was significantly associated with mortality in non-elderly patients $(p=0.031)$ but not in overall patients $(p=$ 0.143 ) and elderly patients $(p=0.456)$ in multivariate logistic regression analysis. The AUC (area under the ROC curve) value of TnT in overall, non-elderly and elderly patients was $0.644,0.693$ and 0.632 , respectively. Combining TnT with GCS increased the sensitivity of predicting mortality of both non-elderly and elderly TBI patients.

\section{Conclusion}

The prognostic value of TnT differed between elderly and non-elderly TBI patients. Level of TnT was associated with mortality of non-elderly TBI patients but not elderly patients. Combining the TnT with GCS could increase the sensitivity of prognosis evaluation.

\section{Introduction}


Estimated occurring nearly sixty-nine million times each year globally, traumatic brain injury (TBI) brings enormous burden to social economics and families of casualties[1]. The high mortality of TBI patients are attributable to complex injury pathophysiology caused by initial external mechanical forces and subsequent secondary brain injury[2]. In addition to intracranial injury, non-neurological organ dysfunction is also commonly observed and has been confirmed associated with outcome of TBI patients[3,4]. One study reported that $22.6 \%$ of TBI patients would develop at least one non-neurological complication during hospitalization[5]. One of the most common non-neurological complications is cardiac injury, which was reported occurring in $22.3 \%$ of isolated severe TBI patients[6]. And it has been verified that cardiac dysfunction was positively correlated with brain injury severity and reduced inhospital survival in moderate to severe TBI patients[7].

Some indicators of cardiac dysfunction such as cardiac troponin, abnormalities of echocardiography and electrocardiogram have been explored and utilized in various clinical settings. It has been discovered that elevation of serum Tnl (Troponin I) level was not only observed in patients with myocardial infarction or acute coronary syndrome, but also the patients diagnosed with sepsis, chronic renal failure, pulmonary embolism or non-traumatic brain injury[8-12]. Previous studies exploring the prognostic value of troponin I (TnI) have found that increased TnI was associated with injury severity and adverse outcome of TBI patients [13-15]. Compared with Tnl negative group, Tnl positive group had longer length of hospital stay, higher Modified Rankin Scale and lower Glasgow Outcome Scale[16]. However, one of these studies concluded that TnI was an effective predictor of mortality in TBI patients under 65 years old but not in those over 65 years old. Another two studies have indicated that TnT (Troponin T) was also valuable in predicting mortality of TBI patients even after adjusting confounders $[17,18]$. However, the most of included participants in these two studies were young TBI patients. We suspected that age might also modify the effect of TnT on outcome of TBI patients. Therefore, we designed this study to explore the prognostic value of TnT in different age subgroups including those younger than 65 and over 65 years old.

\section{Materials And Methods Patients included}

This retrospective observational study was conducted utilizing data from the prospective Multiparameter Intelligent Monitoring in Intensive Care Database III (MIMIC-III database).

Patients admitted to ICUs (Intensive Care Unit) of Beth Israel Deaconess Medical Center between 2001 and 2012 were included in this large critical care database. This freely available database was approved by the Institutional Review Boards of Beth Israel Deaconess Medical Center and the Massachusetts Institute of Technology (MIT). Patients included in this public database were deidentified and anonymized for protecting their privacy. We got the certification to utilize data of MIMIC III after finishing the National Institutes of Health $(\mathrm{NIH})$ Web-based training course and the Protecting Human Research Participants examination. In this study, patients diagnosed with TBI were eligible for this study. There 


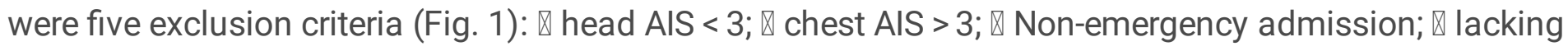

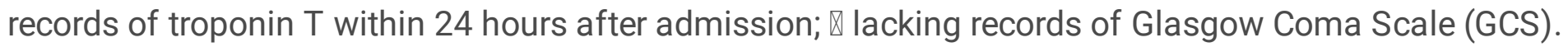
Finally, a total of 520 patients were included in this study.

\section{Data Included}

All collected variables including age, sex, history of underlying disease, records of vital signs on admission., GCS, brain injury types, records of operation and blood transfusion, level of troponin T, length of hospital stay were extracted by us using Navicat Premium 12 (PremiumSoft, Hong Kong). The primary outcome of this study was in-hospital mortality.

\section{Statistical analysis}

The normality of included variables was validated by Kolmogorov-Smirnov tests. All included continuous variables were shown as median (interquartile range) because of the non-normality. Mann-Whitney $U$ test was performed to compare differences between two groups of non-normally distributed continuous variables. And categorical variables were shown as numbers (percentage). The difference between two groups of categorical variables was analyzed by chi-square test. For subgroup analysis, overall patients were divided into two groups based on whether their age $\geq 65$ years old. Univariate logistic regression was performed to find potential risk factors for in-hospital mortality in overall patients and two subgroups. Risk factors with $p<0.05$ were eligible for subsequent multivariate logistic regression analysis. To analyze the real effect of troponin $T$ on outcome of overall patients and two subgroups, the confounding effect of aforementioned risk factors was adjusted by multivariate logistic regression analysis. And odds ratio (OR) and 95\% confidence intervals (Cl) of troponin T in overall patients and two subgroups were calculated and presented. Receiver operating characteristic (ROC) curves were drawn to evaluate the predictive value of troponin T and GCS. Z test was used to compare predictive value between single factors (troponin T or GCS) and combination of factors (troponin T and GCS).

A P value $<0.05$ was considered being of statistical significance. We used SPSS 22.0 Windows software (SPSS, Inc, Chicago, IL) for all statistical analyses and figures drawing.

\section{Results}

\section{Baseline characteristics of included TBI patients}

A total of $520 \mathrm{TBI}$ patients was included in this study. There were 415 survivors and 105 non-survivors with mortality rate of $20.2 \%$ (Table 1). The median age of overall patients was 80 years. There was no significant difference about age between survivors and non-survivors ( 79 vs $81, p=0.217$ ). The male ratio also did not differ between these two groups ( $54 \%$ vs $52.4 \%, p=0.770)$. The percentage of underlying disease including hypertension, coronary heart disease and diabetes mellitus did not differ between survivors and non-survivors. However, non-survivors were more likely to be complicated with previous 
acute myocardial infarction than survivors $(11.4 \%$ vs $4.8 \%, p=0.019)$. Results of vital signs on admission showed no significant difference between survivors and non-survivors. And non-survivors had higher incidence of pupillary no-reaction whether one or both sides ( $9.5 \%$ vs $3.9 \%, p=0.028 ; 6.7 \%$ vs $1.7 \%, p=$ 0.011 ). Furthermore, the GCS of non-survivors was lower than survivors with statistical significance ( $6 \mathrm{vs}$ $14, p<0.001)$. The occurrence rate of several injury patterns including concussion, contusion, laceration, epidural hematoma, subdural hematoma and intracerebral hematoma did not differ between survivors and non-survivors. Whereas non-survivors had significantly higher occurrence rate of subarachnoid hemorrhage than survivors ( $34.3 \%$ vs $24.6 \%, p=0.048)$. The incidence of surgical operation and blood transfusion was higher in non-survivors than survivors without statistical significance $(28.6 \% \mathrm{vs} 24.8 \%, p$ $=0.435 ; 40.0 \%$ vs $38.3 \%, p=0.752$ ). During hospitalization, non-survivors were more likely to suffer arrhythmia than survivors ( $48 \%$ vs $22.3 \%, p<0.001$ ). A remarkable finding was that non-survivors had significantly higher level of troponin $T$ than survivors $(0.02$ vs $0, p<0.001)$. Compared with survivors, nonsurvivors had longer length of ICU stay ( 4 vs $2, p=0.001)$. Instead, the length of hospital stay was shorter in non-survivors than survivors ( 6 vs $8, p<0.001)$. 
Table 1

Baseline characteristics of overall patients and subgroups divided based on survival outcome

\begin{tabular}{|c|c|c|c|c|}
\hline Variables & $\begin{array}{l}\text { Total patients } \\
(\mathrm{N}=520)\end{array}$ & $\begin{array}{l}\text { Survivors }(n=415 \text {, } \\
79.8 \% \text { ) }\end{array}$ & $\begin{array}{l}\text { Non-survivors }(n= \\
105,20.2 \%)\end{array}$ & $\mathbf{p}$ \\
\hline Age (years) & $80(67-86)$ & $79(67-85)$ & $81(67-88)$ & 0.217 \\
\hline Male gender & $279(53.7 \%)$ & $224(54 \%)$ & $55(52.4 \%)$ & 0.770 \\
\hline \multicolumn{5}{|l|}{$\begin{array}{l}\text { History of underlying } \\
\text { disease }\end{array}$} \\
\hline Hypertension & $328(63.1 \%)$ & $260(62.7 \%)$ & $68(64.8 \%)$ & 0.688 \\
\hline Coronary heart Disease & $130(25 \%)$ & $96(23.1 \%)$ & $34(32.4 \%)$ & 0.056 \\
\hline $\begin{array}{l}\text { Acute myocardial } \\
\text { infarction }\end{array}$ & $32(6.2 \%)$ & $20(4.8 \%)$ & $12(11.4 \%)$ & 0.019 \\
\hline Diabetes mellitus & $131(25.2 \%)$ & $100(24.1 \%)$ & 31 (29.5\%) & 0.259 \\
\hline \multicolumn{5}{|l|}{$\begin{array}{l}\text { Vital signs on } \\
\text { admission }\end{array}$} \\
\hline $\begin{array}{l}\text { Systolic blood pressure } \\
(\mathrm{mmHg})\end{array}$ & 139 (127-148) & $139(128-147)$ & $139(124-153)$ & 0.713 \\
\hline $\begin{array}{l}\text { Diastolic blood pressure } \\
(\mathrm{mmHg})\end{array}$ & $62(58-72)$ & $62(59-71)$ & $62(52-73)$ & 0.411 \\
\hline Heart rate $\left(s^{-1}\right)$ & $81(70-93)$ & $81(71-92)$ & $81(70-94)$ & 0.840 \\
\hline Respiratory rate $\left(s^{-1}\right)$ & $18(15-21)$ & $18(15-21)$ & $18(15-21)$ & 0.659 \\
\hline Body temperature $\left({ }^{\circ} \mathrm{C}\right)$ & $36.7(36.2-37.3)$ & $36.7(36.2-37.3)$ & 36.7 (35.9-37.3) & 0.097 \\
\hline $\mathrm{SpO}_{2}(\%)$ & $98(97-100)$ & $98(96.9-100)$ & $99(97.3-100)$ & 0.003 \\
\hline \multicolumn{5}{|l|}{ Pupillary reactivity } \\
\hline No-reaction (one side) & $26(5 \%)$ & $16(3.9 \%)$ & $10(9.5 \%)$ & 0.028 \\
\hline No-reaction (both sides) & $14(2.7 \%)$ & $7(1.7 \%)$ & $7(6.7 \%)$ & 0.011 \\
\hline GCS on admission & $13(7-15)$ & $14(9-15)$ & $6(4-10)$ & $<0.001$ \\
\hline \multicolumn{5}{|l|}{ Injury types } \\
\hline Concussion & $11(2.1 \%)$ & $11(2.7 \%)$ & $0(0)$ & 0.132 \\
\hline Contusion & $15(2.9 \%)$ & $13(3.1 \%)$ & $2(1.9 \%)$ & 0.746 \\
\hline Laceration & 75 (14.4\%) & $64(15.4 \%)$ & $11(10.5 \%)$ & 0.183 \\
\hline Epidural hematoma & $14(2.7 \%)$ & $11(2.7 \%)$ & $3(2.9 \%)$ & 1.000 \\
\hline
\end{tabular}




\begin{tabular}{|c|c|c|c|c|}
\hline Variables & $\begin{array}{l}\text { Total patients } \\
(\mathrm{N}=520)\end{array}$ & $\begin{array}{l}\text { Survivors }(n=415 \text {, } \\
79.8 \% \text { ) }\end{array}$ & $\begin{array}{l}\text { Non-survivors }(n= \\
105,20.2 \%)\end{array}$ & $\mathrm{p}$ \\
\hline Subdural hematoma & $273(52.5 \%)$ & $223(53.7 \%)$ & $50(47.6 \%)$ & 0.263 \\
\hline $\begin{array}{l}\text { Subarachnoid } \\
\text { hemorrhage }\end{array}$ & $138(26.5 \%)$ & $102(24.6 \%)$ & $36(34.3 \%)$ & 0.048 \\
\hline Intracerebral hematoma & $68(13.1 \%)$ & $48(11.6 \%)$ & $20(19 \%)$ & 0.051 \\
\hline Operation & $133(25.6 \%)$ & $103(24.8 \%)$ & $30(28.6 \%)$ & 0.435 \\
\hline Blood transfusion & $201(38.7 \%)$ & $159(38.3 \%)$ & $42(40.0 \%)$ & 0.752 \\
\hline $\begin{array}{l}\text { Arrhythmia during } \\
\text { hospitalization }\end{array}$ & $221(42.5 \%)$ & $169(40.7 \%)$ & $52(49.5 \%)$ & 0.105 \\
\hline Troponin T $(\mu \mathrm{g} / \mathrm{L})$ & $0.01(0-0.03)$ & $0(0-0.03)$ & $0.02(0-0.07)$ & $\begin{array}{l}<.001 \\
0.001\end{array}$ \\
\hline $\begin{array}{l}\text { Length of ICU stay } \\
\text { (days) }\end{array}$ & $2(1-5)$ & $2(1-4)$ & $4(12-7)$ & 0.001 \\
\hline $\begin{array}{l}\text { Length of hospital stay } \\
\text { (days) }\end{array}$ & $7(4-13)$ & $8(5-14)$ & $6(3-11)$ & $<0.001$ \\
\hline
\end{tabular}

Compared with non-elderly TBI patients, elderly TBI patients were more commonly complicated with underlying diseases including hypertension $(p<0.001)$, coronary heart disease $(p<0.001)$ and diabetes mellitus ( $p=0.038$ ) (Table 2$)$. The systolic blood pressure (139 vs $135, p=0.023$ ) was higher in elderly patients whereas diastolic blood pressure ( 62 vs $71, p<0.001)$, heart rate $(80$ vs $89, p<0.001)$ and body temperature ( 36.7 vs $36.9, p=0.002$ ) were all lower in elderly patients. Elderly patients had significantly higher GCS than non-elderly patients ( $14 \mathrm{vs} 10, p=0.016$ ). And elderly patients were more likely to receive blood transfusion ( $(41.9 \%$ vs $26.8 \%, p=0.003)$ and suffer arrhythmia ( $48 \%$ vs $22.3 \%, p<0.001)$ during hospitalization. Moreover, the serum level of TnT was higher in elderly patients than non-elderly ( $0.01 \mathrm{vs}$ $0, p=0.014)$. While the in-hospital mortality did not significantly differ between elderly and non-elderly patients $(20.3 \%$ vs $19.6 \%, p=0.870)$. 
Table 2

Baseline characteristics of non-elderly and elderly patients

\begin{tabular}{|c|c|c|c|}
\hline Variables & Non-elderly $(n=112,21.5 \%)$ & Elderly $(n=408,78.5 \%)$ & p \\
\hline Age & $56(47-61)$ & $82(77-88)$ & $<0.001$ \\
\hline Male gender & $85(75.9 \%)$ & $194(47.5 \%)$ & $<0.001$ \\
\hline \multicolumn{4}{|l|}{ History of underlying disease } \\
\hline Hypertension & 49 (43.8\%) & $279(68.4 \%)$ & $<0.001$ \\
\hline Coronary heart Disease & $8(7.1 \%)$ & $122(29.9 \%)$ & $<0.001$ \\
\hline Acute myocardial infarction & $3(2.7 \%)$ & $29(7.1 \%)$ & 0.060 \\
\hline Diabetes mellitus & $20(17.9 \%)$ & $111(27.2 \%)$ & 0.038 \\
\hline \multicolumn{4}{|l|}{ Vital signs on admission } \\
\hline Systolic blood pressure & $135(123-146)$ & $139(129-149)$ & 0.023 \\
\hline Diastolic blood pressure & $71(62-79)$ & $62(56-69)$ & $<0.001$ \\
\hline Heart rate & $89(77-103)$ & $80(68-90)$ & $<0.001$ \\
\hline Respiratory rate & $18(14-21)$ & $18(15-21)$ & 0.918 \\
\hline Body temperature & $36.9(36.3-37.7)$ & $36.7(36.1-37.2)$ & 0.002 \\
\hline $\mathrm{SpO}_{2}(\%)$ & $98.5(97-100)$ & $98(96.9-100)$ & 0.012 \\
\hline \multicolumn{4}{|l|}{ Pupillary reactivity } \\
\hline No-reaction (one side) & $4(3.6 \%)$ & $22(5.4 \%)$ & 0.416 \\
\hline No-reaction (both sides) & $3(2.7 \%)$ & $11(2.7 \%)$ & 1.000 \\
\hline GCS on admission & $10(6-15)$ & $14(8-15)$ & 0.016 \\
\hline \multicolumn{4}{|l|}{ Injury types } \\
\hline Concussion & $8(7.1 \%)$ & $3(0.7 \%)$ & $<0.001$ \\
\hline Contusion & $4(3.6 \%)$ & $11(2.7 \%)$ & 0.541 \\
\hline Laceration & $24(21.4 \%)$ & $51(12.5 \%)$ & 0.022 \\
\hline Epidural hematoma & $4(3.6 \%)$ & $10(2.5 \%)$ & 0.513 \\
\hline Subdural hematoma & $46(41.1 \%)$ & $227(55.6 \%)$ & 0.006 \\
\hline Subarachnoid hemorrhage & $30(26.8 \%)$ & $108(26.5 \%)$ & 0.947 \\
\hline Intracerebral hematoma & $16(14.3 \%)$ & $52(12.7 \%)$ & 0.671 \\
\hline
\end{tabular}




\begin{tabular}{|llll|}
\hline Variables & Non-elderly $(\mathbf{n = 1 1 2 , 2 1 . 5 \% )}$ & Elderly $(\mathbf{n = 4 0 8 , 7 8 . 5 \% )}$ & $\mathbf{p}$ \\
\hline Operation & $23(20.5 \%)$ & $110(27 \%)$ & 0.160 \\
\hline Blood transfusion & $30(26.8 \%)$ & $171(41.9 \%)$ & 0.003 \\
\hline $\begin{array}{l}\text { Arrhythmia during } \\
\text { hospitalization }\end{array}$ & $25(22.3 \%)$ & $196(48 \%)$ & $<0.001$ \\
\hline Troponin T & $0(0-0.01)$ & $0.01(0-0.04)$ & 0.014 \\
\hline Mortality & $22(19.6 \%)$ & $83(20.3 \%)$ & 0.870 \\
\hline Length of ICU stay (days) & $3(2-6)$ & $2(1-5)$ & 0.113 \\
\hline Length of hospital stay (days) & $7(3-14)$ & $7(5-13)$ & 0.155 \\
\hline $\mathrm{SpO}_{2}$ Oxygen saturation, GCS Glasgow Coma Scale & & \\
\hline
\end{tabular}

\section{Univariate Logistic Regression Analysis Of Risk Factors For Mortality}

In overall included patients, history of acute myocardial infarction $(O R=2.548, p=0.015)$, pupil noreaction $(O R=2.805, p<0.001)$, subarachnoid hemorrhage $(O R=1.601, p=0.045)$, intracerebral hematoma $(O R=1.799, p=0.044)$ and troponin $T(O R=2.541, p=0.025)$ were positively associated poor outcome (Table 3$)$. While body temperature $(O R=0.735, p=0.011)$ and GCS $(O R=0.770, p<0.001)$ were inversely correlated with poor outcome. In non-elderly patients, only pupil no-reaction $(O R=4.839 . p<$ $0.001)$ and GCS $(O R=0.723 . p<0.001)$ were found related with in-hospital mortality (Table 4). And in elderly patients, coronary heart disease $(O R=1.869, p=0.014)$, body temperature $(O R=0.692, p=0.012)$, pupil no-reaction $(\mathrm{OR}=2.398, \mathrm{p}<0.001)$, GCS $(\mathrm{OR}=0.772, \mathrm{p}<0.001)$ and intracerebral hematoma $(\mathrm{OR}=$ $1.917, p=0.048)$ and arrhythmia $(O R=1.640, p=0.047)$ were significantly correlated with in-hospital mortality (Table 5). 
Table 3

Univariate analysis of risk factors for mortality in overall patients

\begin{tabular}{|llll|}
\hline Variables & OR & $\mathbf{9 5 \%}$ & $\mathbf{P}$ \\
\hline age & 1.004 & $0.989-1.019$ & 0.576 \\
\hline Male gender & 0.938 & $0.611-1.440$ & 0.770 \\
\hline Hypertension & 1.096 & $0.701-1.713$ & 0.689 \\
\hline Coronary heart Disease & 1.591 & $0.996-2.541$ & 0.052 \\
\hline Acute myocardial infarction & 2.548 & $1.203-5.398$ & 0.015 \\
\hline Diabetes mellitus & 1.320 & $0.820-2.124$ & 0.253 \\
\hline Systolic blood pressure & 1.000 & $0.993-1.006$ & 0.912 \\
\hline Diastolic blood pressure & 0.996 & $0.984-1.008$ & 0.491 \\
\hline Heart rate & 0.998 & $0.986-1.010$ & 0.769 \\
\hline Respiratory rate & 0.992 & $0.950-1.035$ & 0.703 \\
\hline Body temperature & 0.735 & $0.581-0.931$ & 0.011 \\
\hline SpO2 (\%) & 0.987 & $0.935-1.041$ & 0.623 \\
\hline Pupil no-reaction & 2.805 & $2.052-3.836$ & $<0.001$ \\
\hline GCS & 0.770 & $0.728-0.815$ & $<0.001$ \\
\hline Concussion & 1.428 & $0.929-2.195$ & 0.104 \\
\hline Contusion & 2.541 & $1.123-5.752$ & 0.025 \\
\hline Laceration & 1.0 .001 & - & 0.999 \\
\hline Epidural hematoma & 1.601 & $1.010-2.538$ & 0.045 \\
\hline Subdural hematoma & 0.600 & $0.133-2.703$ & 0.506 \\
\hline Subarachnoid hemorrhage & 0.642 & $0.325-1.266$ & 0.200 \\
\hline Intracerebral hematoma & 1.080 & $0.296-3.944$ & 0.907 \\
\hline Operation & 0.783 & $0.510-1.202$ & 0.263 \\
\hline Blood transfusion & $1.015-3.189$ & 0.044 \\
\hline Arrhythmia & $0.751-1.955$ & 0.432 \\
\hline Troponin T & $0.693-1.663$ & 0.751 \\
\hline OR Odds ratio, Cl confidence interval, Sp & & & \\
\hline
\end{tabular}


Table 4

Univariate analysis of risk factors for mortality in non-elderly patients

\begin{tabular}{|c|c|c|c|}
\hline Variables & OR & $95 \% \mathrm{Cl}$ & $\mathbf{p}$ \\
\hline age & 0.986 & $0.949-1.025$ & 0.487 \\
\hline Male gender & 2.303 & $0.625-8.486$ & 0.210 \\
\hline Hypertension & 0.865 & $0.336-2.229$ & 0.765 \\
\hline Coronary heart Disease & $<0.001$ & - & 0.999 \\
\hline Acute myocardial infarction & 8.9 & $0.769-103.029$ & 0.080 \\
\hline Diabetes mellitus & 1.471 & $0.470-4.602$ & 0.508 \\
\hline Systolic blood pressure & 0.995 & $0.982-1.008$ & 0.459 \\
\hline Diastolic blood pressure & 0.993 & $0.970-1.015$ & 0.519 \\
\hline Heart rate & 0.999 & $0.976-1.023$ & 0.948 \\
\hline Respiratory rate & 1.005 & $0.923-1.094$ & 0.909 \\
\hline Body temperature & 0.834 & $0.547-1.272$ & 0.400 \\
\hline SpO2 (\%) & 1.108 & $0.860-1.427$ & 0.428 \\
\hline Pupil no-reaction & 4.839 & $2.429-9.641$ & $<0.001$ \\
\hline GCS & 0.723 & $0.621-0.842$ & $<0.001$ \\
\hline Concussion & $<0.001$ & - & 0.999 \\
\hline Contusion & $<0.001$ & - & 0.999 \\
\hline Laceration & 1.099 & $0.359-3.363$ & 0.868 \\
\hline Epidural hematoma & 4.400 & $0.584-33.142$ & 0.150 \\
\hline Subdural hematoma & 1.250 & $0.489-3.197$ & 0.641 \\
\hline Subarachnoid hemorrhage & 1.359 & $0.493-3.749$ & 0.553 \\
\hline Intracerebral hematoma & 1.444 & $0.417-5.003$ & 0.562 \\
\hline Operation & 2.158 & $0.757-6.151$ & 0.150 \\
\hline Blood transfusion & 1.359 & $0.493-3.749$ & 0.553 \\
\hline Arrhythmia & 0.730 & $0.222-2.396$ & 0.604 \\
\hline Troponin T & 2.228 & $0.791-6.278$ & 0.130 \\
\hline
\end{tabular}


Table 5

Univariate analysis of risk factors for mortality in elderly patients

\begin{tabular}{|c|c|c|c|}
\hline Variables & OR & $95 \% \mathrm{Cl}$ & $\mathbf{p}$ \\
\hline age & 1.027 & $0.992-1.062$ & 0.128 \\
\hline Male gender & 0.81 & $0.498-1.316$ & 0.394 \\
\hline Hypertension & 1.173 & $0.692-1.990$ & 0.553 \\
\hline Coronary heart Disease & 1.869 & $1.132-3.085$ & 0.014 \\
\hline Acute myocardial infarction & 2.206 & $0.984-4.945$ & 0.055 \\
\hline Diabetes mellitus & 1.288 & $0.761-2.179$ & 0.345 \\
\hline Systolic blood pressure & 1.001 & $0.993-1.009$ & 0.777 \\
\hline Diastolic blood pressure & 0.997 & $0.983-1.011$ & 0.708 \\
\hline Heart rate & 0.998 & $0.984-1.012$ & 0.787 \\
\hline Respiratory rate & 0.987 & $0.939-1.038$ & 0.619 \\
\hline Body temperature & 0.692 & $0.519-0.922$ & 0.012 \\
\hline SpO2 (\%) & 0.981 & $0.929-1.036$ & 0.488 \\
\hline Pupil no-reaction & 2.398 & $1.673-3.437$ & $<0.001$ \\
\hline GCS & 0.772 & $0.725-0.823$ & $<0.001$ \\
\hline Concussion & $<0.001$ & - & 0.999 \\
\hline Contusion & 0.867 & $0.184-4.091$ & 0.857 \\
\hline Laceration & 0.485 & $0.199-1.179$ & 0.110 \\
\hline Epidural hematoma & 0.428 & $0.053-3.428$ & 0.424 \\
\hline Subdural hematoma & 0.686 & $0.423-1.113$ & 0.127 \\
\hline Subarachnoid hemorrhage & 1.672 & $0.997-2.806$ & 0.051 \\
\hline Intracerebral hematoma & 1.917 & $1.005-3.658$ & 0.048 \\
\hline Operation & 1.049 & $0.611-1.799$ & 0.863 \\
\hline Blood transfusion & 1.013 & $0.622-1.651$ & 0.958 \\
\hline Arrhythmia & 1.640 & $1.008-2.670$ & 0.047 \\
\hline Troponin T & 3.168 & $0.902-11.124$ & 0.072 \\
\hline
\end{tabular}




\section{Association Between Troponin T And Outcome After Adjusting Confounders}

To verify the independent association between troponin $\mathrm{T}$ and outcome, multivariate logistic regression analyses were performed in overall patients and two subgroups (age $<65$, age $\geq 65$ ). After adjusting the confounding effects of acute myocardial infarction, body temperature, pupil no-reaction, GCS, subarachnoid hemorrhage, intracerebral hematoma, the OR of troponin T was 1.909 without statistical significance $(p=0.143)$ in overall included patients (Table 6$)$. While in patients $<65$ years old, the OR of troponin T was 3.178 with statistical significance $(p=0.040)$ after adjusting the confounding effects of pupil no-reaction, GCS. However, level of troponin T was not significantly associated with outcome in patients whose age $\geq 65(O R=1.839, p=0.456)$, after considering the effects of confounders including coronary heart disease, body temperature, pupil no-reaction, GCS, intracerebral hematoma and arrhythmia.

Table 6

Association between troponin $\mathrm{T}$ and mortality after adjusting confounders in overall patients and subgroups

\begin{tabular}{|c|c|c|c|}
\hline & OR & $95 \% \mathrm{Cl}$ & p \\
\hline Overall patients* & 1.909 & $0.803-4.536$ & 0.143 \\
\hline$<65$ years old** & 3.178 & $1.057-9.562$ & 0.040 \\
\hline$\geq 65$ years old ${ }^{\#}$ & 1.839 & $0.371-9.113$ & 0.456 \\
\hline \multicolumn{4}{|c|}{$\begin{array}{l}\text { * adjusted for acute myocardial infarction, body temperature, pupil no-reaction, GCS, subarachnoid } \\
\text { hemorrhage, intracerebral hematoma. }\end{array}$} \\
\hline \multicolumn{4}{|c|}{ 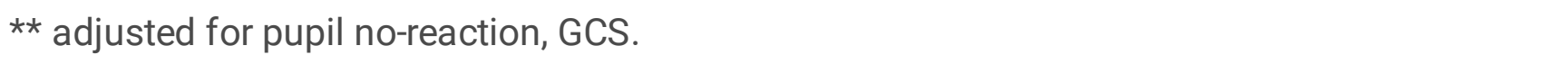 } \\
\hline \multicolumn{4}{|c|}{$\begin{array}{l}\text { \# adjusted for coronary heart disease, body temperature, pupil no-reaction, GCS, intracerebral } \\
\text { hematoma, arrhythmia. }\end{array}$} \\
\hline \multicolumn{4}{|c|}{ OR Odds ratio, $\mathrm{Cl}$ confidence interval } \\
\hline
\end{tabular}

\section{Prognostic value of troponin $\mathrm{T}$ in overall patients and subgroups}

The AUC value of troponin T and GCS for predicting mortality of overall patients was 0.644 and 0.794 , respectively (Table 7) (Fig. 2A). GCS had significantly higher AUC value than troponin $T(Z=3.6939, p<$ 0.05). Combining troponin T could not improve the predictive value of single assessment of GCS ( 0.814 vs $0.794, Z=0.6006, p>0.05)$. For those age $<65$, the AUC value of troponin T and GCS was 0.693 and 0.829 , respectively (Fig. $2 B)$. Troponin had comparable AUC value with GCS $(Z=1.5804, p>0.05)$. The AUC value of combining troponin T with GCS was 0.862 , which was higher than single GCS, though without statistical significance $(Z=0.4568, p>0.05)$. However, the AUC value of combining troponin $T$ with GCS was significantly higher than single evaluation of troponin $T(Z=2.0505, p<0.05)$. For those 
age $\geq 65$, the AUC value of troponin T was 0.632 (Fig. 2C), which was significantly lower than 0.793 of GCS $(Z=3.5778, p<0.05)$. Combining troponin T with GCS could not improve the predictive value of single GCS $(Z=0.5260, p>0.05)$. A remarkable discovery was that combining troponin $T$ could distinctly improve the sensitivity of predicting mortality.

Table 7

Prognostic value of troponin T and GCS in overall patients and subgroups

\begin{tabular}{|lllll|}
\hline \multicolumn{1}{|c}{ AUC } & $95 \% \mathrm{Cl}$ & Sensitivity & Specificity \\
\hline Overall patients & & & & \\
\hline TnT & 0.644 & $0.582-0.706$ & 0.590 & 0.687 \\
GCS & 0.794 & $0.746-0.842$ & 0.747 & 0.733 \\
GCS + TnT & 0.814 & $0.770-0.858$ & 0.867 & 0.617 \\
\hline Patients $<65$ years old & & & & \\
\hline TnT & 0.693 & $0.562-0.824$ & 0.500 & 0.833 \\
GCS & 0.829 & $0.723-0.934$ & 0.833 & 0.818 \\
\hline GCS + TnT & 0.862 & $0.768-0.955$ & 0.864 & 0.822 \\
\hline Patients $\geq 65$ years old & & & & 0.646 \\
\hline TnT & 0.632 & $0.562-0.703$ & 0.614 & 0.699 \\
\hline GCS & 0.793 & $0.739-0.847$ & 0.778 & 0.652 \\
\hline GCS + TnT & 0.812 & $0.764-0.860$ & 0.855 & troponin T, \\
\hline $\begin{array}{l}\text { AUC area under the receiver operating characteristics curve, Cl confidence interval, TnT } \\
\text { GCS Glasgow Coma Scale }\end{array}$ & & & \\
\hline
\end{tabular}

\section{Discussion}

In our study, non-survivors had significantly higher serum TnT level than survivors. Results of univariate logistic regression showed TnT was only statistically significant in overall patients but not two age subgroups. However, the real effect of TnT on outcome of TBI patients should be evaluated after adjusting potential confounders. Actually, we found that TnT was only statistically significant in subgroup of patients younger than 65 by performing multivariate logistic regression analysis. And TnT was more effective in predicting in-hospital mortality of non-elderly TBI patients with a relatively high AUC value. This result was similar with previous finding that $\mathrm{Tnl}$ was an useful biological prognostic marker in isolated severe TBI patients whose age $\leq 65$ but not patients whose age $>65[14]$.

There are three isoforms of troponin complex: troponin C, I, and T[19]. The characteristics of troponin C isoform existing in both cardiac and skeletal muscle makes it not be considered as a sensitive marker of 
cardiac injury. However, the myocardium specific troponin I and $\mathrm{T}$ is an useful biomarker for myocardial necrosis and has been clinically used for early diagnosis and prognosis in patients with cardiopulmonary diseases such as acute coronary syndrome, acute myocardial infarction, heart failure and pulmonary embolism[20-27]. Whereas the clinical use of cardiac troponin could not only be limited in primary cardiopulmonary diseases. In fact, the serum cardiac troponin level could reflect the severity of developing cardiovascular complications involved in non-cardiovascular system specific disease such as sepsis and trauma[28-30]. The association between increased serum cardiac troponin level and poor outcome of these diseases has also been confirmed in previous studies[31-33]. In addition, the elevation of cardiac troponin was also discovered in non-traumatic brain injury patients including ischemic stroke, intracerebral hemorrhage and subarachnoid hemorrhage[34-37]. And the degree of increased cardiac troponin was positively correlated with occurrence of cardiovascular dysfunction, brain injury severity and poor outcome of these brain injured patients[11, 38-43]. Previous studies also concluded that increased Tnl level after TBI was related with poor outcome of these patients $[13,14]$. The increase of cardiac troponin after various forms of brain injury was mainly attributable to the neurogenic stunned myocardium, presenting with reversible left ventricular systolic dysfunction, pulmonary edema and cardiogenic shock, which was likely caused by sympathetic hyperactivity induced excessive release of catecholamines and acute inflammatory response[39, 44-47].

The development of cardiac dysfunction has been verified as a risk factor for in-hospital mortality of TBI patients[6]. It has been reported in previous studies that the incidence of cardiac dysfunction after TBI ranged from $13-22.3 \%[6,48-50]$. These studies usually evaluated the cardiac function according to the signs of echocardiogram such as reduced left ventricular ejection fraction and regional wall motion abnormalities. A novel neurogenic cardiac injury score (NCIS), which was calculated based on rising troponin I, abnormal echocardiography and hypotension, was recently designed and confirmed independently associated with in-hospital mortality of patients with severe head trauma after adjusting confounders[51]. Previous studies have investigated the prognostic value of troponin I in TBI patients and found that effect of troponin I on outcome could be modified by age[13-16]. The TnI was not as valuable in predicting outcome in elderly TBI patients as in non-elderly patients[14]. Another two studies discovered that high sensitive troponin T (HsTnT) was also an effective prognostic indicator in TBI patients $[17,18]$. However, the patients included in these two studies were from the same trauma database and mostly were young injured patients with median age of thirties. The homogeneous population of these studies limited the general applicability of TnT in TBI patients. Our study specially investigated the association between TnT and outcome of TBI patients in different age subgroups. The TnT level was independently associated in-hospital mortality of included non-elderly TBI patients but not elderly TBI patients. And single assessment of TnT performed well in predicting outcome of non-elderly TBI patients with relatively high AUC. Instead, the low AUC, specificity and sensitivity of TnT in elderly TBI patients indicated that it could not be considered as an accurate indicator of prognosis in this age subgroup. This finding emphasized the conclusion that age could modify the effect of both TnI and TnT on outcome of TBI patients. The limited effect of TnT on outcome of elderly TBI patients were likely explained by the elevation of baseline TnT caused by natural aging and complicated chronic 
cardiovascular disease. More detailed mechanism involved in this finding should be explored in future study. Meanwhile, more sensitive cardiac injury biomarkers in TBI patients should be developed to effectively detect potential cardiopulmonary complications and evaluate prognosis of elderly TBI patients. One noteworthy finding in this study was that TnT combined with GCS significantly improved the sensitivity of predicting in-hospital mortality in both age subgroups, whether AUC was statistically improved or not. Based on this discovery, we concluded that assessment of TnT for evaluating cardiac injury was beneficial for physicians to screen patients with high probabilities of poor prognosis and give intensive care and therapies protecting cardiac function such as beta-blockers in early stage.

There were several limitations in this study. Firstly, we did not evaluate serial or peak level of TnT during hospitalization for included patients. This limitation could confound the effect of TnT on outcome of TBI patients. Secondly, we could not compare the predictive value between $\mathrm{Tnl}$ and $\mathrm{TnT}$ due to the incomplete records of Tnl in included patients, Thirdly, included patients mainly were elderly, with only 112 nonelderly patients. However, previous studies have analyzed the association between TnT and outcome of TBI patients with younger age. Finally, some patients without records of TnT were excluded from this study, which could lead to selection bias. Therefore, our results should be verified in further prospective study.

\section{Conclusion}

The prognostic value of TnT in elderly and non-elderly TBI patients is different. Higher level of TnT indicates increased mortality of non-elderly TBI patients. Evaluating the TnT is beneficial for physicians to predict outcome of TBI patients with high sensitivity.

\section{Declarations}

\section{Acknowledgements}

None

\section{Authors' contributions}

RRW collected clinical data and performed statistical analysis of the data. RRW and MH were major contributor in writing the manuscript. YK revised the manuscript. All authors have read and approved the manuscript.

\section{Funding}

This study was financially supported by the 1.3 .5 project for disciplines of excellence-Clinical Research Incubation Project, West China Hospital, Sichuan University (19HXFH012).

\section{Availability of data and materials}


The datasets used and/or analyzed during the current study are unavailable because of the ethical requirements of the public critical care database MIMIC III.

\section{Ethics approval and consent to participate}

The study was performed using data from the public database MIMIC III. This freely available database was approved by the Institutional Review Boards of Beth Israel Deaconess Medical Center and the Massachusetts Institute of Technology (MIT).Patients included in this public database were deidentified and anonymized for protecting their privacy.

\section{Consent for publication}

Not applicable.

\section{Competing interests}

The authors declare that they have no competing interests.

\section{References}

1. Dewan MC, Rattani A, Gupta S, Baticulon RE, Hung YC, Punchak M, et al. Estimating the global incidence of traumatic brain injury. Journal of neurosurgery. 2018:1-18; doi:10.3171/2017.10.Jns17352.

2. Menon DK, Schwab K, Wright DW, Maas Al. Position statement: definition of traumatic brain injury. Arch Phys Med Rehabil. 2010;91 11:1637-40. doi:10.1016/j.apmr.2010.05.017.

3. Zygun D. Non-neurological organ dysfunction in neurocritical care: impact on outcome and etiological considerations. Curr Opin Crit Care. 2005;11 2:139-43. doi:10.1097/01.ccx.0000155356.86241.c0.

4. Corral L, Javierre CF, Ventura JL, Marcos P, Herrero JI, Manez R. Impact of non-neurological complications in severe traumatic brain injury outcome. Crit Care (London England). 2012;16(2):R44. doi:10.1186/cc11243.

5. Omar M, Moore L, Lauzier F, Tardif PA, Dufresne P, Boutin A, et al. Complications following hospital admission for traumatic brain injury: A multicenter cohort study. J Crit Care. 2017;41:1-8. doi:10.1016/j.jcrc.2017.04.031.

6. Prathep S, Sharma D, Hallman M, Joffe A, Krishnamoorthy V, Mackensen GB, et al. Preliminary report on cardiac dysfunction after isolated traumatic brain injury. Critical care medicine. 2014;42 1:142-7. doi:10.1097/CCM.0b013e318298a890.

7. Gibbons PW, Goldberg RJ, Muehlschlegel S. A pilot study evaluating a simple cardiac dysfunction score to predict complications and survival among critically-ill patients with traumatic brain injury. $J$ Crit Care. 2019;54:130-5. doi:10.1016/j.jcrc.2019.08.017. 
8. Babuin L, Jaffe AS. Troponin: the biomarker of choice for the detection of cardiac injury. CMAJ: Canadian Medical Association journal = journal de l'Association medicale canadienne. 2005;173 10:1191-202; doi: 10.1503/cmaj/051291.

9. Hamm CW, Giannitsis E, Katus HA. Cardiac troponin elevations in patients without acute coronary syndrome. Circulation. 2002;106 23:2871-2. doi:10.1161/01.cir.0000044342.50593.63.

10. Jeremias A, Gibson CM. Narrative review: alternative causes for elevated cardiac troponin levels when acute coronary syndromes are excluded. Ann Intern Med. 2005;142 9:786-91. doi:10.7326/0003-4819-142-9-200505030-00015.

11. Hays A, Diringer MN. Elevated troponin levels are associated with higher mortality following intracerebral hemorrhage. Neurology. 2006;66 9:1330-4. doi:10.1212/01.wnl.0000210523.22944.9b.

12. Roongsritong $\mathrm{C}$, Warraich I, Bradley C. Common causes of troponin elevations in the absence of acute myocardial infarction: incidence and clinical significance. Chest. 2004;125 5:1877-84. doi:10.1378/chest.125.5.1877.

13. Salim A, Hadjizacharia P, Brown C, Inaba K, Teixeira PG, Chan L, et al. Significance of troponin elevation after severe traumatic brain injury. The Journal of trauma. 2008;64 1:46-52. doi:10.1097/TA.0b013e31815eb15a.

14. Cai SS, Bonds BW, Hu PF, Stein DM. The role of cardiac troponin I in prognostication of patients with isolated severe traumatic brain injury. The journal of trauma acute care surgery. 2016;80(3):477-83. doi:10.1097/ta.0000000000000916.

15. Rimaz S, Ashraf A, Marzban S, Haghighi M, Zia Ziabari SM, Biazar G, et al. Significance of Cardiac Troponin I Elevation in Traumatic Brain Injury Patients. Anesthesiology pain medicine. 2019;9(2):e90858. doi:10.5812/aapm.90858.

16. Bender M, Stein M, Schoof B, Kolodziej MA, Uhl E, Schöller K. Troponin I as an early biomarker of cardiopulmonary parameters during the first $24 \mathrm{~h}$ of intensive care unit treatment in isolated traumatic brain injury patients. Injury. 2020;51 5:1189-95. doi:10.1016/j.injury.2020.01.002.

17. El-Menyar A, Asim M, Ramzee AF, Nabir S, Ahmed MN, Al-Thani A, et al. Bio-Shock Index: Proposal and Rationale for a New Predictive Tool for In-Hospital Mortality in Patients with Traumatic Brain Injury. World neurosurgery. 2019;132:e169-e77. doi:10.1016/j.wneu.2019.08.229.

18. El-Menyar A, Asim M, Latifi R, Bangdiwala SI, Al-Thani H. Predictive value of positive high-sensitivity troponin $T$ in intubated traumatic brain injury patients. Journal of neurosurgery. 2018;129 6:1541-9. doi:10.3171/2017.7.Jns17675.

19. Zhang L, Wang Z, Qi S. Cardiac Troponin Elevation and Outcome after Subarachnoid Hemorrhage: A Systematic Review and Meta-analysis. Journal of stroke cerebrovascular diseases: the official journal of National Stroke Association. 2015;24 10:2375-84.

doi:10.1016/j.jstrokecerebrovasdis.2015.06.030.

20. Reichlin T, Twerenbold R, Reiter M, Steuer S, Bassetti S, Balmelli C, et al. Introduction of highsensitivity troponin assays: impact on myocardial infarction incidence and prognosis. Am J Med. 2012;125 12:1205-13.e1. doi:10.1016/j.amjmed.2012.07.015. 
21. Potocki M, Reichlin T, Thalmann S, Zellweger C, Twerenbold R, Reiter M, et al. Diagnostic and prognostic impact of copeptin and high-sensitivity cardiac troponin $T$ in patients with pre-existing coronary artery disease and suspected acute myocardial infarction. Heart. 2012;98 7:558-65. doi:10.1136/heartjnl-2011-301269.

22. Reiter M, Twerenbold R, Reichlin T, Benz B, Haaf P, Meissner J, et al. Early diagnosis of acute myocardial infarction in patients with pre-existing coronary artery disease using more sensitive cardiac troponin assays. European heart journal. 2012;33 8:988-97. doi:10.1093/eurheartj/ehr376.

23. Fathil MF, Md Arshad MK, Gopinath SC, Hashim U, Adzhri R, Ayub RM, et al. Diagnostics on acute myocardial infarction: Cardiac troponin biomarkers. Biosens Bioelectron. 2015;70:209-20. doi:10.1016/j.bios.2015.03.037.

24. Árnadóttir Á, Vestergaard KR, Sölétormos G, Steffensen R, Goetze JP, Iversen K. Prediction of coronary heart disease or heart failure using high-sensitivity cardiac troponin T: A pilot study. Eur $\mathrm{J}$ Clin Invest. 2018;48 10:e13009. doi:10.1111/eci.13009.

25. Adamson PD, Anderson JA, Brook RD, Calverley PMA, Celli BR, Cowans NJ, et al. Cardiac Troponin I and Cardiovascular Risk in Patients With Chronic Obstructive Pulmonary Disease. J Am Coll Cardiol. 2018;72 10:1126-37. doi:10.1016/j.jacc.2018.06.051.

26. Ng AC, Yong AS, Chow V, Chung T, Freedman SB, Kritharides L. Cardiac troponin-T and the prediction of acute and long-term mortality after acute pulmonary embolism. Int J Cardiol. 2013;165(1):12633. doi:10.1016/j.ijcard.2011.07.107.

27. Kucher N, Wallmann D, Carone A, Windecker S, Meier B, Hess OM. Incremental prognostic value of troponin I and echocardiography in patients with acute pulmonary embolism. European heart journal. 2003;24 18:1651-6. doi:10.1016/s0195-668x(03)00394-4.

28. Landesberg G, Jaffe AS, Gilon D, Levin PD, Goodman S, Abu-Baih A, et al. Troponin elevation in severe sepsis and septic shock: the role of left ventricular diastolic dysfunction and right ventricular dilatation*. Critical care medicine. 2014;42 4:790-800. doi:10.1097/ccm.0000000000000107.

29. Edouard AR, Felten ML, Hebert JL, Cosson C, Martin L, Benhamou D. Incidence and significance of cardiac troponin I release in severe trauma patients. Anesthesiology. 2004;101 6:1262-8. doi:10.1097/00000542-200412000-00004.

30. Rajan GP, Zellweger R. Cardiac troponin I as a predictor of arrhythmia and ventricular dysfunction in trauma patients with myocardial contusion. The Journal of trauma. 2004;57 4:801-8. doi:10.1097/01.ta.0000135157.93649.72. discussion 8.

31. Vallabhajosyula S, Sakhuja A, Geske JB, Kumar M, Poterucha JT, Kashyap R, et al. Role of Admission Troponin-T and Serial Troponin-T Testing in Predicting Outcomes in Severe Sepsis and Septic Shock. Journal of the American Heart Association. 2017;6:9. doi:10.1161/jaha.117.005930.

32. Decavèle M, Gault N, Gauss T, Pease S, Moyer JD, Paugam-Burtz C, et al. Cardiac troponin I as an early prognosis biomarker after trauma: a retrospective cohort study. Br J Anaesth. 2018;120 6:1158-64. doi:10.1016/j.bja.2018.03.010. 
33. Braun CK, Schaffer A, Weber B, Huber-Lang M, Kalbitz M, Preßmar J. The Prognostic Value of Troponin in Pediatric Polytrauma. Front Pead. 2019;7:477. doi:10.3389/fped.2019.00477.

34. Peddada K, Cruz-Flores S, Goldstein LB, Feen E, Kennedy KF, Heuring T, et al. Ischemic Stroke with Troponin Elevation: Patient Characteristics, Resource Utilization and In-Hospital Outcomes. Cerebrovascular diseases (Basel, Switzerland). 2016;42 3-4:213 - 23; doi: 10.1159/000445526.

35. VanHouten J, Fricker G, Collins B, Bhatia R, Ellis C, Schrag M. Circulating Troponin I Level in Patients with Acute Ischemic Stroke. Curr Neurol Neurosci Rep. 2018;18 6:32. doi:10.1007/s11910-018-08426.

36. Sandhu R, Aronow WS, Rajdev A, Sukhija R, Amin H, D'Aquila K, et al. Relation of cardiac troponin I levels with in-hospital mortality in patients with ischemic stroke, intracerebral hemorrhage, and subarachnoid hemorrhage. The American journal of cardiology. 2008;102 5:632-4. doi:10.1016/j.amjcard.2008.04.036.

37. Xu M, Lin J, Wang D, Liu M, Hao Z, Lei C. Cardiac troponin and cerebral herniation in acute intracerebral hemorrhage. Brain behavior. 2017;7 6:e00697. doi:10.1002/brb3.697.

38. Yaghi S, Chang AD, Ricci BA, Jayaraman MV, McTaggart RA, Hemendinger M, et al. Early Elevated Troponin Levels After Ischemic Stroke Suggests a Cardioembolic Source. Stroke. 2018;49(1):121-6. doi:10.1161/strokeaha.117.019395.

39. Naidech AM, Kreiter KT, Janjua N, Ostapkovich ND, Parra A, Commichau C, et al. Cardiac troponin elevation, cardiovascular morbidity, and outcome after subarachnoid hemorrhage. Circulation. 2005;112 18:2851-6. doi:10.1161/circulationaha.105.533620.

40. Duello KM, Nagel JP, Thomas CS, Blackshear JL, Freeman WD. Relationship of Troponin T and Ageand Sex-Adjusted BNP Elevation Following Subarachnoid Hemorrhage with 30-Day Mortality. Neurocritical care. 2015;23 1:59-65. doi:10.1007/s12028-014-0105-6.

41. He Y, Liu Q, Wang J, Wang DW, Ding H, Wang W. Prognostic value of elevated cardiac troponin I in patients with intracerebral hemorrhage. Clinical cardiology. 2020;43 4:338-45. doi:10.1002/clc.23320.

42. Alkhachroum AM, Miller B, Chami T, Tatsuoka C, Sila C. A troponin study on patients with ischemic stroke, intracerebral hemorrhage and subarachnoid hemorrhage: Type II myocardial infarction is significantly associated with stroke severity, discharge disposition and mortality. Journal of clinical neuroscience: official journal of the Neurosurgical Society of Australasia. 2019;64:83-8. doi:10.1016/j.jocn.2019.04.005.

43. Gerner ST, Auerbeck K, Sprügel MI, Sembill JA, Madžar D, Gölitz P, et al. Peak Troponin I Levels Are Associated with Functional Outcome in Intracerebral Hemorrhage. Cerebrovascular diseases (Basel, Switzerland). 2018;461-2:72-81; doi: 10.1159/000492395.

44. Hinson HE, Sheth KN. Manifestations of the hyperadrenergic state after acute brain injury. Curr Opin Crit Care. 2012;18 2:139-45. doi:10.1097/MCC.0b013e3283513290.

45. El-Menyar A, Goyal A, Latifı R, Al-Thani H, Frishman W. Brain-Heart Interactions in Traumatic Brain Injury. Cardiol Rev. 2017;25 6:279-88. doi:10.1097/crd.0000000000000167. 
46. Lambert E, Du XJ, Percy E, Lambert G. Cardiac response to norepinephrine and sympathetic nerve stimulation following experimental subarachnoid hemorrhage. Journal of the neurological sciences. 2002;198(1-2):43-50. doi:10.1016/s0022-510x(02)00073-4.

47. Kenigsberg BB, Barnett CF, Mai JC, Chang JJ. Neurogenic Stunned Myocardium in Severe Neurological Injury. Curr Neurol Neurosci Rep. 2019;19 11:90. doi:10.1007/s11910-019-0999-7.

48. Venkata C, Kasal J. Cardiac Dysfunction in Adult Patients with Traumatic Brain Injury: A Prospective Cohort Study. Clin Med Res. 2018;16(3-4):57-65. doi:10.3121/cmr.2018.1437.

49. Krishnamoorthy V, Rowhani-Rahbar A, Chaikittisilpa N, Gibbons EF, Rivara FP, Temkin NR, et al. Association of Early Hemodynamic Profile and the Development of Systolic Dysfunction Following Traumatic Brain Injury. Neurocritical care. 2017;26 3:379-87. doi:10.1007/s12028-016-0335-x.

50. Krishnamoorthy V, Rowhani-Rahbar A, Gibbons EF, Rivara FP, Temkin NR, Pontius C, et al. Early Systolic Dysfunction Following Traumatic Brain Injury: A Cohort Study. Critical care medicine. 2017;45 6:1028-36. doi:10.1097/ccm.0000000000002404.

51. Hasanin A, Kamal A, Amin S, Zakaria D, El Sayed R, Mahmoud K, et al. Incidence and outcome of cardiac injury in patients with severe head trauma. Scand J Trauma Resusc Emerg Med. 2016;24:58. doi:10.1186/s13049-016-0246-z.

\section{Figures}




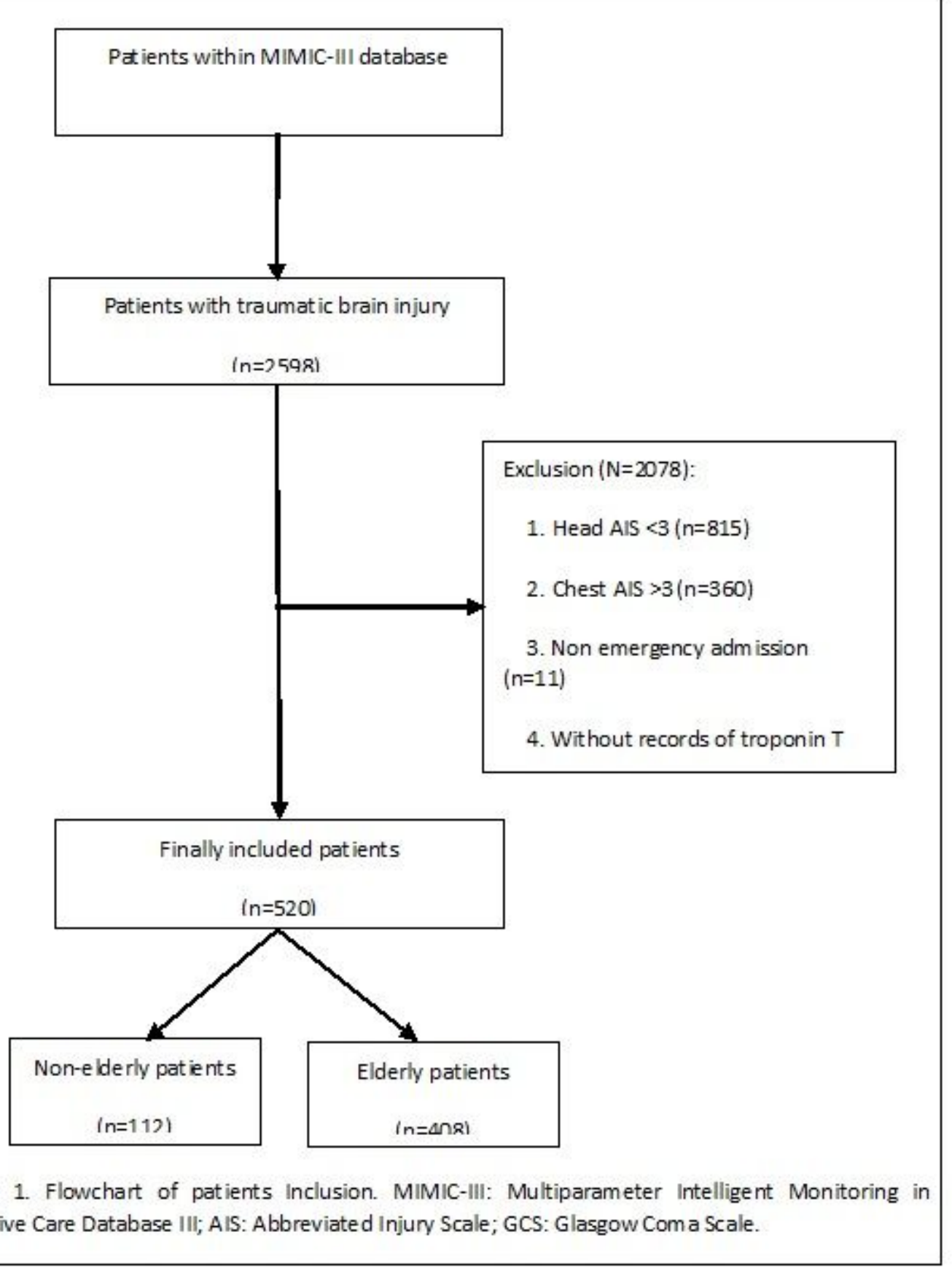

\section{Figure 1}

Figure 1 


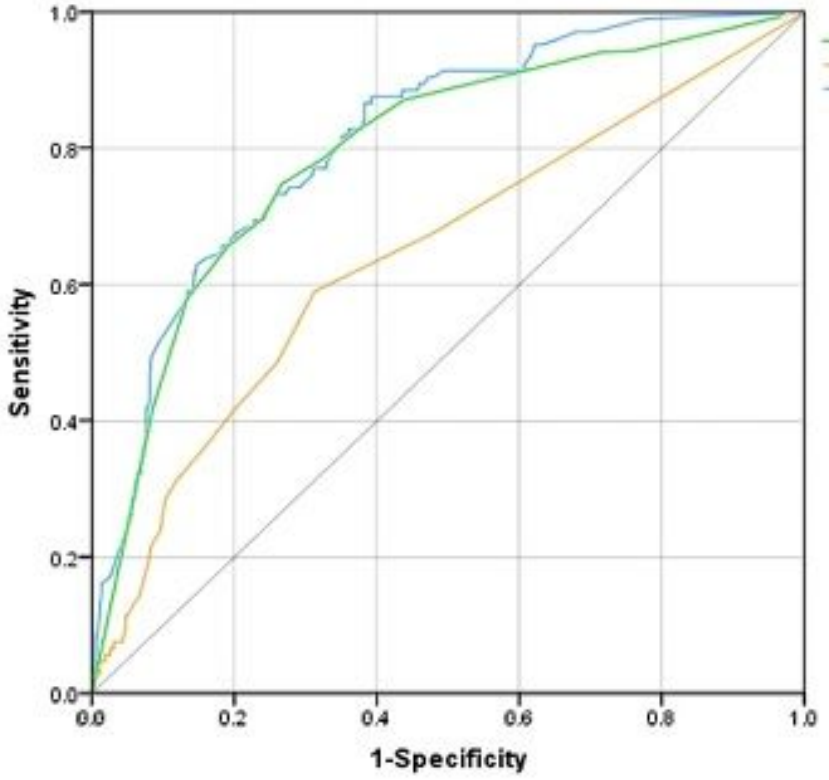

Figure 2A. ROC curves of GCS, troponin T and combination of these twe fartnre in inclurled nuerall TRI natients

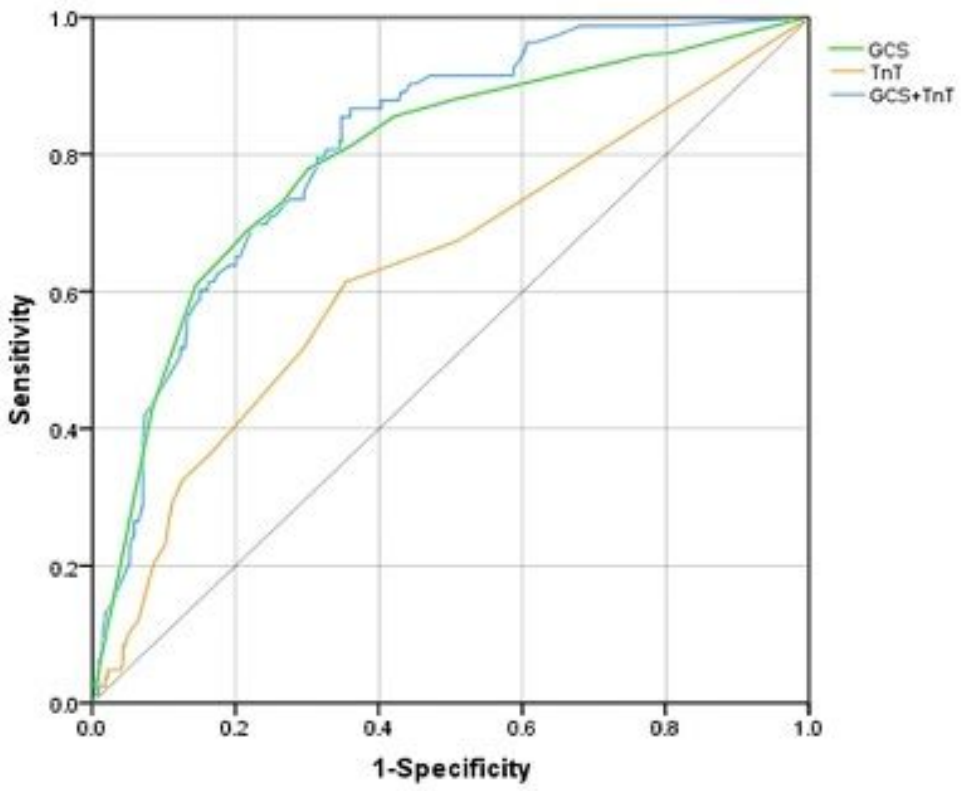

Figure 2C. ROC curves of GCS, troponin T and combination of these two factors in included elderlv TBI patients

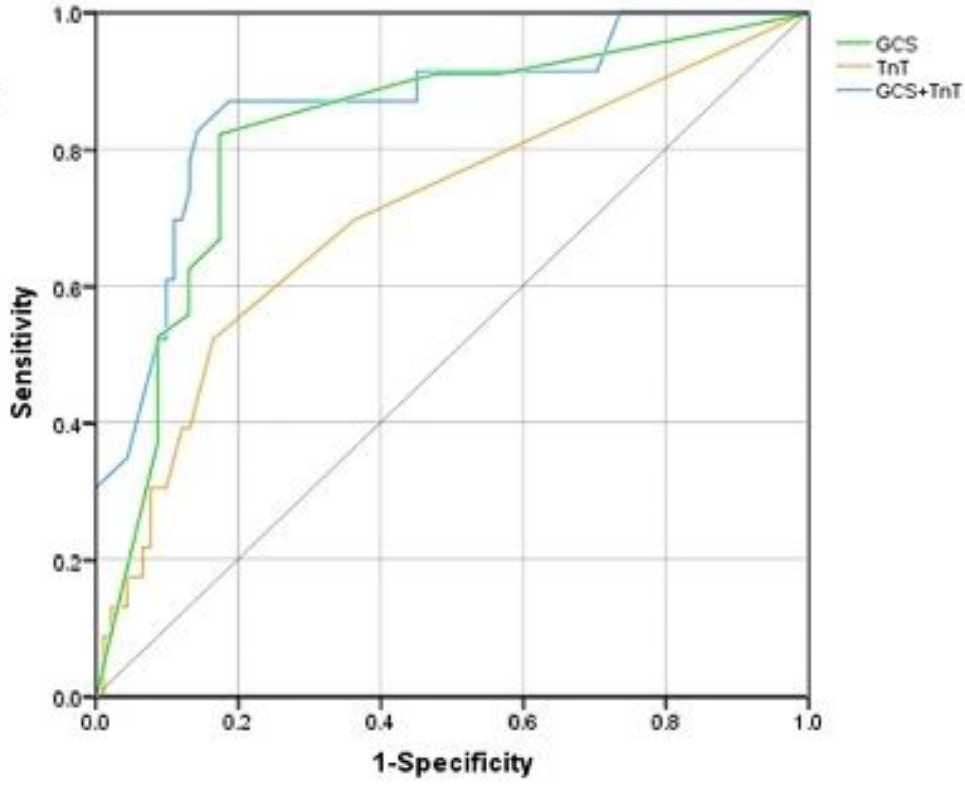

Figure $2 \mathrm{~B}$. ROC curves of GCS, troponin $\mathrm{T}$ and combination of these two factors in included non-elderly TBI patients

\section{Figure 2}

Figure 2 